\title{
Fork Method Stabilization of Fiber Embedded Ceramics for TEM Observation
}

\author{
Stephen W. Ordway
}

Department of Physics at University of Missouri Saint Louis, One University Blvd., Saint Louis, MO, 63121 USA

Materials are embedded with fibers made of a stronger material in order to provide support and stability. The original material is strengthened along the length of the fibers and is more durable. In creating a TEM specimen to look end-on at the SiC fibers in a $\mathrm{ZrB}_{2}$ matrix, we discovered the process of cutting the fiber makes the sample friable. This necessitated development of a sample preparation method that would provide support to the fiber embedded sample for end-on observation.

In the past, friable samples would be prepared by a method in which the sample would be mounted in a support medium and placed in a copper or brass tube [1]. This process strengthens the material, allowing slices to be cut for creating discs that could then be abraded, polished, and milled for TEM observation. Typically this process uses copper or brass tubing and the medium used to fill the tube varies. Example of media in the literature include filling the tubing with epoxy, and sliding the sample in between molybdenum tines and sliding this set up into the tubing [2]. Such methods have been used to give tensile strength to inhomogeneous samples.

To stabilize our fiber embedded material, the sample was prepared using a copper tube with an outer diameter of $3 \mathrm{~mm}$ and a stainless steel rod that was cut as described in Figure 1, which we referred to as the fork method. Other sources described this as a reinforced cross-sectional method. The sample was abraded with $30 \mu \mathrm{m}$ diamond lapping paper to dimensions of $1 \mathrm{~mm} \times 1.5 \mathrm{~mm}$. The length of the sample is equal to the length of the slot; normally $10-15 \mathrm{~mm}$. The fibers in the sample were oriented to run the length of the slot. Once the sample was fitted snugly in the stainless steel fork, all edges of the sample, the inner and outer part of the fork, and the copper tube were coated with Vishay M-bond 610. The pieces were assembled before the epoxy dried, with the sample fitting snugly into the steel fork and the copper tubing being slid on with some tension to hold the apparatus together. Disc shaped slices 300$500 \mu \mathrm{m}$ thick were then abraded, polished, dimpled, and milled. The final product was a sample offering an end-on view of the embedded fibers as seen in Figure 2.

$30 \mu \mathrm{m} \mathrm{SiC} \mathrm{lapping} \mathrm{paper} \mathrm{removed} 8 \mu \mathrm{m} / 5 \mathrm{~min}$ from the $\mathrm{ZrB}_{2}$ samples and $2 \mu \mathrm{m} / 5 \mathrm{~min}$ from the $\mathrm{ZrB}{ }_{2}$ with SiC. After switching to $30 \mu \mathrm{m}$ diamond lapping paper, there was little variation in abrasion rates among $\mathrm{ZrB}_{2}, \mathrm{ZrB}_{2}$ and $\mathrm{SiC}$, and $\mathrm{ZrB}_{2}$ with embedded fibers. All of these samples abraded approximately $100 \mu \mathrm{m} / 5 \mathrm{~min}$ with variation based on the number of samples the sheet had abraded.

When creating the samples, about 2 of 3 samples were successfully abraded to fit into the steel fork and copper tubing. Our first fork sample had 4 failed discs cut before one remained in tact. The method was modified so the epoxy cured in a vacuum oven to vacuum impregnate the sample. Only one disc cut off of this failed, before a useable sample was created. Of the successful samples, $50 \%$ of them held together through a number of TEM observations. Damaged discs resulted from the steel support falling out due to inadequate use of epoxy. Regarding useful thin area, the perforation typically had a $200 \mu \mathrm{m}$ circumference with a $5 \mu \mathrm{m}$ border giving about $1000 \mu \mathrm{m}^{2}$ of useful area. Some of the samples had issue with material falling out, resulting in a number of holes after milling; most excess holes had no useful 
thin area. In the future, mounting the sample with extra pressure being exerted from the steel fork might prevent this.

No variation in abrasion rates of $\mathrm{ZrB}_{2}$ and reinforced $\mathrm{ZrB}_{2}$ with fibers prior to mounting the sample in the steel support was observed. Without the support however, it was found impossible to create a fiber embedded sample with traditional planar section methods. The fork method created a buffer around the sample and compression to reduce the likelihood of the sample falling apart. By reinforcing the sample with a malleable material such as copper, the sample was more durable. The use of stainless steel was chosen, as its milling rate is much lower than the $\mathrm{ZrB}_{2}$ with embedded fibers. Therefore, the steel support would not be milled away, leaving the sample vulnerable. Mounting the sample in the steel fork and copper tubing allowed for a number of slices to be taken for observation from a single sample.

[1] S. B. Newcomb, C. B. Boothroyd, and W. M. Stobbs, Journal of Microscopy, Vol. 140 (1985), pp 195-207.

[2] D. V. Sridhara Rao, et al. TEM Specimen Techniques, Microscopy: Science, Technology, Applications and Education, (2010) pp 1232-44.

[3] Special Thanks to: Project support from the Air Force contract number FA8650-05-D-5807 and Greg Hilmas at MST; Bob Collins for TEM observation and data; P. Fraundorf for discussion; equipment access and guidance from David Osborn; the UMSL Center for NanoScience; Shuhan Lin at UMSL for original design; Bruce Burkeen for fork creation; and Austin Johnson at MST for Figure 1a.
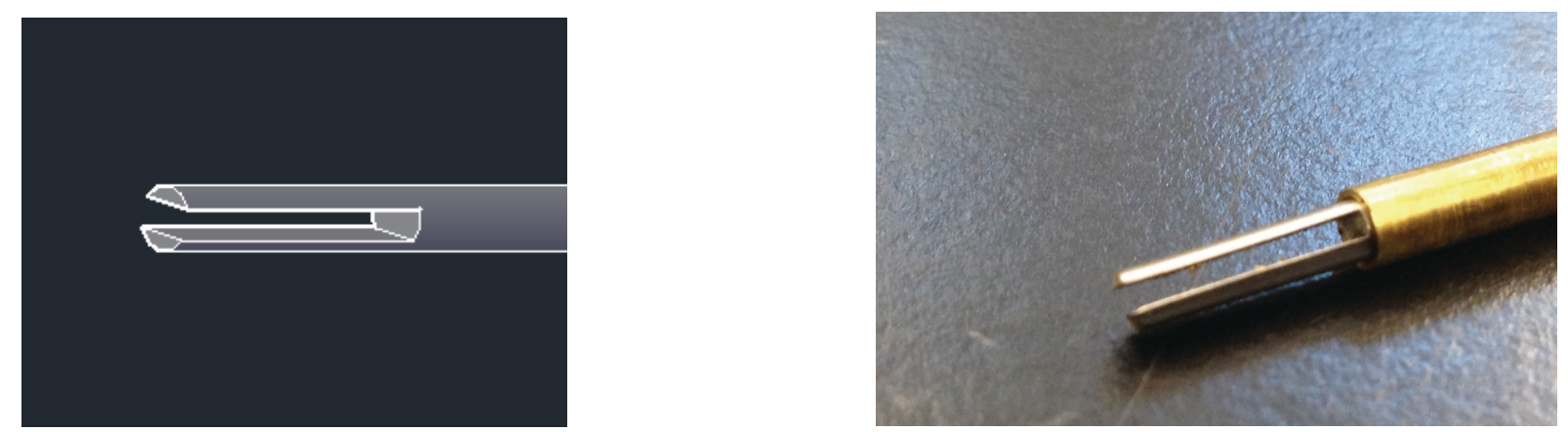

Figure 1. Stainless Steel Rod with $1 \mathrm{~mm}$ slot $10-15 \mathrm{~mm}$ deep for sample to mount in slot (left). Steel fork and Copper tubing (right).
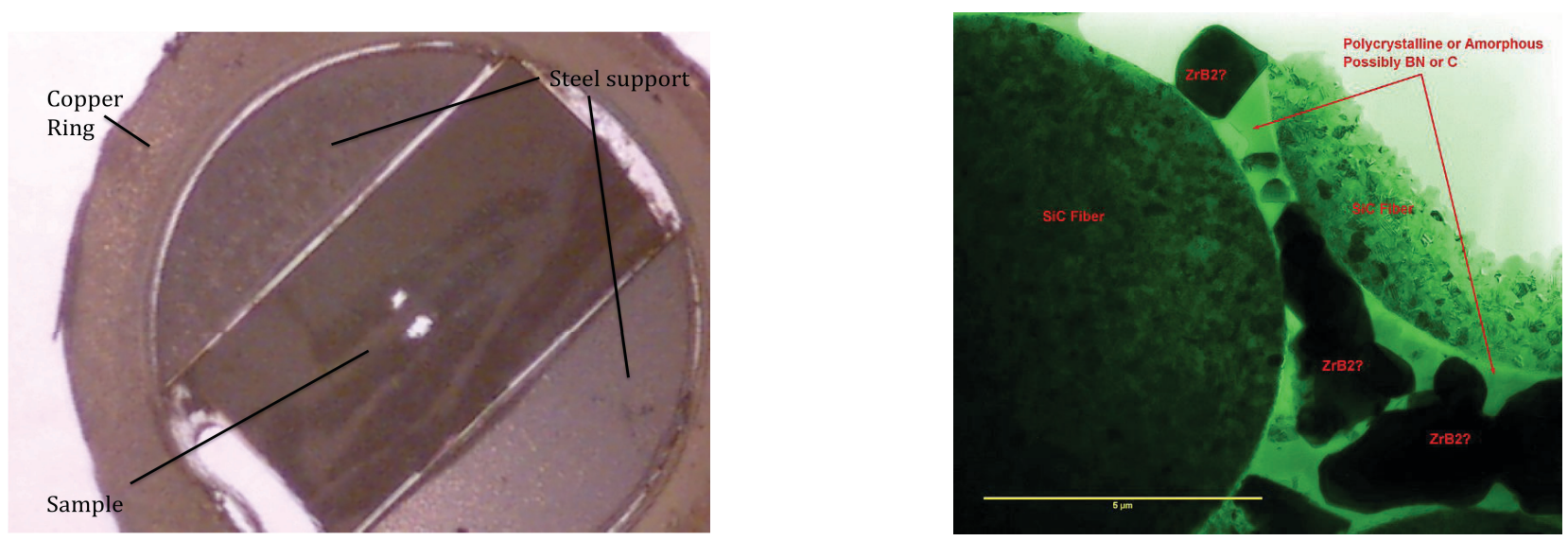

Figure 2. Here is a light image of a finished sample after abrasion, dimpling, and milling (left) and a TEM image of the sample (right). 